\title{
Leiomiosarcoma del cordón espermático: aportación de dos casos
}

\author{
Soto Delgado M, Pedrero Márquez G*, Jiménez Romero ME, Navas Martínez MC. \\ Servicio de Urología. *Unidad de Enfermería. Hospital Universitario de Puerto Real. Cádiz.
}

Actas Urol Esp. 2007;31(8):911-914

\section{RESUMEN}

LEIOMIOSARCOMA DEL CORDÓN ESPERMÁTICO: APORTACIÓN DE DOS CASOS

En los sarcomas paratesticulares, salvo en el rabdomiosarcoma, el único tratamiento factible y resolutivo es el quirúrgico radical. La vía obligatoria e ideal será la inguinotomía, con exéresis del cordón funicular y teste adyacente. Exige controles periódicos a largo plazo, dada las recidivas que pueden ocurrir incluso mucho tiempo después del diagnóstico. Ante una eventual recidiva la cirugía volverá a ser el único arma a nuestro alcance para el control de este tipo tumoral.

Presentamos 2 casos de leiomiosarcoma paratesticular, un tumor de escasa frecuencia.

Palabras clave: Leiomiosarcoma. Cordón espermático. Tratamiento.

\section{ABSTRACT \\ LEIOMYOSARCOMA OF THE SPERMATIC CORD: REPORT OF TWO CASES}

Radical surgery is the only feasible and successful treatment for paratesticular sarcomas, with the exception of rabdomyosarcoma. The inguinal approach is the ideal and obligatory one, including excision of the spermatic cord and adjacent testicle. Long-term periodic follow-up visits are mandatory because relapses may ocurre even long time after diagnosis: In the case of relapse, surgery is again the only tool we have to control this type of tumor.

We present two cases of paratesticular leiomyosarcoma, a tumor un common.

Keywords: Leiomyosarcoma. Spermatic cord. Treatment.

$\mathrm{E}^{\mathrm{s}}$ xiste un conjunto de tumores malignos intraescrotales, que representan un número muy reducido cuando se les compara con las neoplasias testiculares y que nacen de los conductos deferentes, epidídimo, cordón espermático, órganos vestigiales o de las túnicas vaginales. En la mayoría de los casos (90\%) surgen del cordón espermático, aunque en ocasiones resulta difícil comprobarlo. Dado que todos ellos poseen prácticamente una clínica, un pronóstico, una historia natural y un tratamiento muy similar, parece lógico agruparlos bajo una misma denominación: la de neoplasias paratesticulares ${ }^{1}$.

La mayoría de los tumores que ocurren en la región paratesticular son de origen mesenquimatoso siendo la gran mayoría de ellos $(70 \%)$ benignos.
El 30\% restante corresponden a neoplasias malignas. El tumor maligno más frecuente es el rabdomiosarcoma $(40 \%$ de todos los tumores paratesticulares), seguido por el leiomiosarcoma y el liposarcoma. Mientras que el rabdomiosarcoma afecta fundamentalmente a niños y adolescentes, el leiomiosarcoma, el liposarcoma y otros tipos de sarcomas menos comunes ocurren generalmente en adultos ${ }^{2}$.

Debido a que se consideran tumores raros, presentamos en el siguiente trabajo dos casos de leiomiosarcoma de cordón espermático.

\section{Caso clínico 1 \\ MATERIAL Y MÉTODO}

Varón de 75 años de edad con antecedentes personales de hipertensión arterial, diabetes mellitus 
no insulinodependiente, adenomectomía de próstata en 1996 que consulta por tumoración en hemiescroto izquierdo de 1 mes de evolución. A la exploración física se palpaba una masa de consistencia firme, de situación yuxtatesticular pero independiente de éste. La transiluminación fue negativa. En la ecografía escrotal practicada se evidenció una lesión nodular hipoecoica redondeada de 6,2 x 5,3 cm en zona posterior escrotal izquierda que desplazaba teste hacia delante (Fig. 1). Los marcadores tumorales (alfa fetoproteína y beta HCG) fueron normales. Ante estos hallazgos de realizó una orquiectomía radical izquierda con extirpación de la masa por vía inguinal siendo el diagnóstico histopatológico de leiomiosarcoma de cordón espermático que se introduce en algunos puntos en parénquima testicular (Fig. 2).

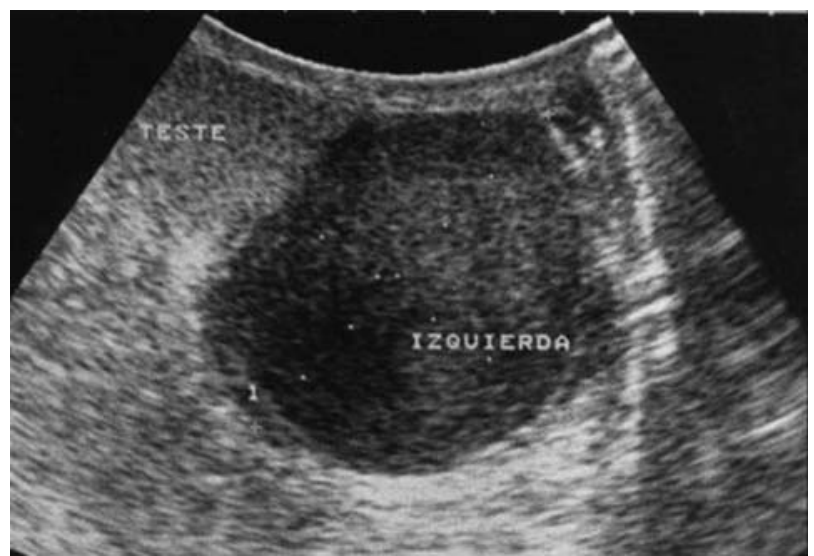

FIGURA 1. Eco escrotal.

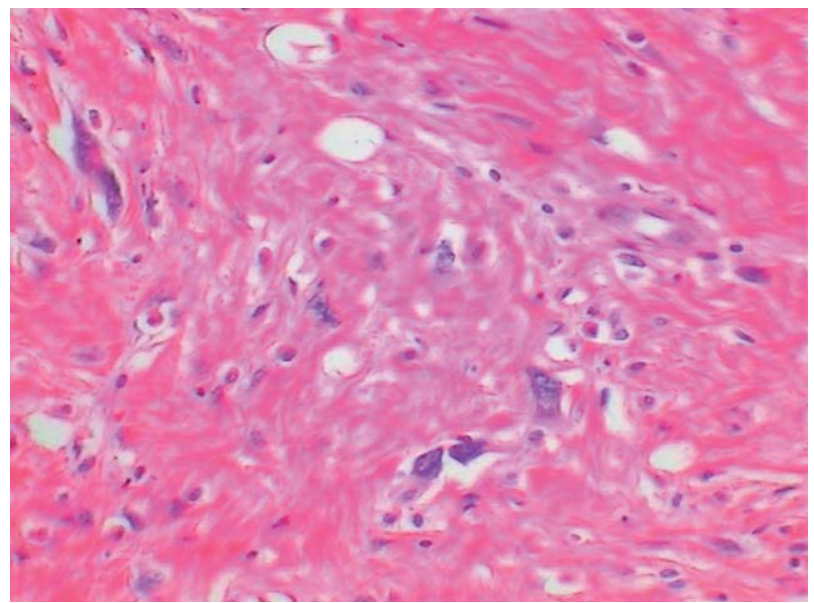

FIGURA 2. Proliferación de células sarcomatosas con marcada atipia y diferenciación hacia músculo liso.
El estudio de extensión realizado (TAC abdominopélvico y radiografía de tórax) no evidenció la presencia de metástasis. Tras 3 años de seguimiento, el paciente presentó una recidiva local y a distancia de su enfermedad lo que provocó su fallecimiento 8 meses después. El paciente y la familia se negaron a recibir tratamiento adyuvante tras la extirpación de la masa inicial así como a recibir cualquier tipo de terapia tras la recidiva.

\section{Caso clínico 2}

Varón de 76 años de edad con antecedentes personales de EPOC, hipertensión arterial, accidente cerebro-vascular con hemiparesia residual y RTU de tumor vesical superficial (pTaGI) que consultó por tumoración en hemiescroto derecho de 2-3 meses de evolución. A la exploración física se palpaba una masa sólida en cordón espermático derecho independiente del testículo de dicho lado. La transiluminación fue negativa así como los marcadores tumorales (alfa fetoproteína y beta HCG). En la ecografia escrotal practicada se observó en la parte superior de hemiescroto derecho una masa sólida de $4 \mathrm{~cm}$ lobulada, bien delimitada, hetrogénea e independiente del testículo (Fig. 3).

Se llevó a cabo una orquiectomía radical derecha con extirpación de la masa por vía inguinal. El diagnóstico histopatológico de la pieza quirúr-

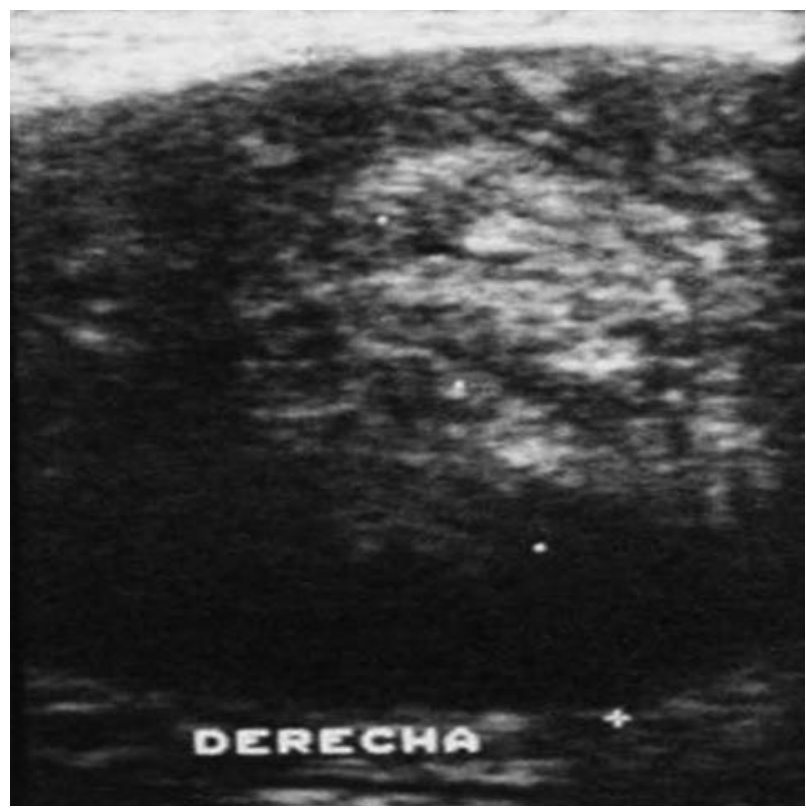

FIGURA 3. Eco escrotal. 
gica fue: Leiomiosarcoma de cordón espermático que no alcanza bordes quirúrgicos ni infiltra parénquima testicular, rete testis, epidídimo ni estructuras vasculares (Fig. 4).

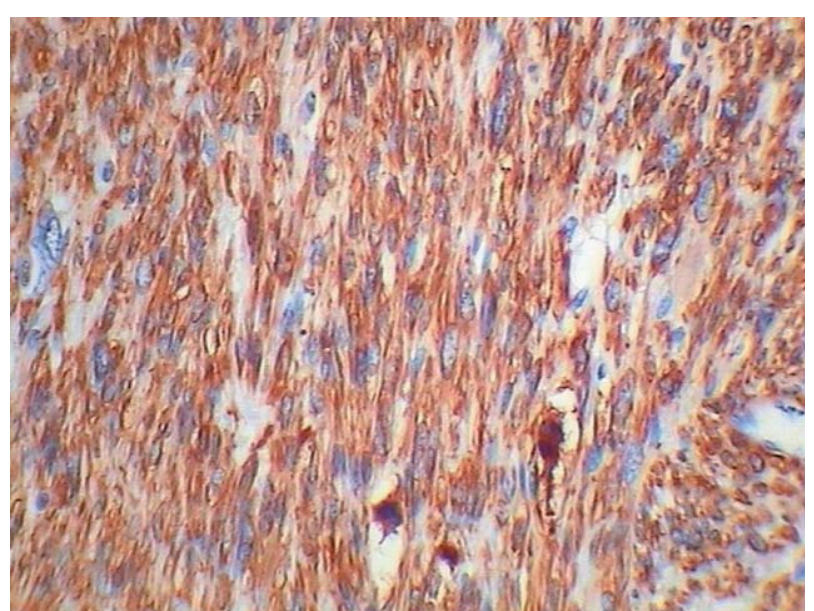

FIGURA 4. Intensa positividad de las células sarcomatosas frente a actina-músculo liso.

El estudio de extensión realizado (TAC abdominopélvico y radiografía de tórax) fue negativo.

No se consideró llevar a cabo ningún tratamiento adyuvante por el mal estado general que presentaba el paciente debido a su patología concomitante. En la actualidad, el paciente se encuentra libre de tumor tras un seguimiento de 24 meses.

\section{DISCUSIÓN}

A la hora de referirnos a las neoplasias intraescrotales comprobamos que existe un conjunto de tumores, que representa un número muy reducido cuando se les compara con las neoplasias testiculares y que reciben la denominación de neoplasias paratesticulares. La incidencia corresponde aproximadamente al 2-3\% $0^{1,3}$.

Desde el punto de vista anatomoclínico las neoplasias paratesticulares se agrupan en benignas y malignas. Las primeras representan el 70\% y dentro de las malignas hay que resaltar que los sarcomas representan más del 90\% ${ }^{1,3,4}$.

Desde un punto de vista clínico-patológico, los sarcomas se clasifican en dos grupos en relación a la edad de presentación y al tipo histológico. En este sentido se distinguen los sarcomas desarrollados en los niños y adolescentes (rabdomiosarcoma) y los que ocurren en la edad adulta (liposarcoma, leiomiosarcoma y fibrosarcoma). El pico de incidencia de los leiomiosarcomas está entre la sexta y la séptima década de la vida, tal y como se ha observado en nuestros dos pacientes 5 .

En relación con el diagnóstico, las neoplasias paratesticulares debutan como una tumoración, que a través de su examen clínico se puede definir el tamaño, la consistencia, la movilidad y la ubicación inguinal, funicular o intraescrotal, separada o no del testículo y/o epidídimo ${ }^{1}$. Con respecto a los pacientes que aportamos, la tumoración no englobaba ni al testículo ni al epidídimo.

Con la ecografía escrotal y con los marcadores tumorales, generalmente negativos, se completaría el estudio local de la tumoración paratesticu$\operatorname{lar}^{2,4}$. En los dos pacientes de nuestro trabajo, los marcadores tumorales (alfa fetoproteína y beta HCG) fueron normales.

El estudio radiológico abdominal mediante TAC o incluso la resonancia magnética nos ayudará en el estadiaje ganglionar y visceral.

El diagnóstico definitivo vendrá dado por el estudio histopatológico de la pieza una vez realizada la orquiectomía radical por vía inguinal con clampeo temprano del cordón para evitar la diseminación de las células tumorales. En aquellos casos en los que el informe histológico nos indique la presencia de márgenes positivos, una nueva cirugía sería obligada, resecando el resto de estructuras adyacentes ${ }^{2}$.

En el caso de los leiomiosarcomas del cordón espermático, no se recomienda una linfadenectomía retroperitoneal ${ }^{1,2,4,5}$.

Es fundamental aportar al diagnóstico el grado de diferenciación histológica de cara a poder plantear tratamiento coadyuvante y establecer un pronóstico. Costa $^{6}$ lo estableció en tres grados:

- Grado 1: no necrosis, menos de 6 mitosis por cada 10 aumentos.

- Grado 2: ocasionales núcleos pleomórficos y necrosis en menos del $15 \%$ del material tumoral con actividad mitótica mayor de 6 por campo.

- Grado 3: mas de 15\% de necrosis sin tener en cuenta el número de mitosis o la morfología nuclear. 
Los leiomiosarcomas del cordón espermático muestran una fuerte propensión a la recidiva local (ocurriendo en la mayoría de los casos en los 3 años posteriores al diagnóstico) y cuando sucede lo hace hacia una forma más indiferenciada. Por ello la exploración rutinaria periódica y la exéresis amplia de cualquier atisbo de recidiva debe ser obligada ${ }^{2,4,5,7-9}$.

No obstante, el pronóstico depende fundamentalmente de la existencia de metástasis que suelen ocurrir por vía hemática. Las metástasis afectan por lo general al pulmón y al cerebro ${ }^{1}$.

Los datos que existen en la literatura sobre el valor de la quimioterapia en los leiomiosarcomas son anecdóticos. En opinión de prácticamente la mayoría de los autores, la quimioterapia sistemática tan sólo mantendrá un papel paliativo en presencia de metástasis viscerales ${ }^{2}$.

No existe uniformidad de criterios a la hora de indicar la necesidad de una terapia adyuvante a la cirugía. A pesar de considerar a este tumor como radiorresistente, algunos autores defienden que la radioterapia local después de la orquiectomía radical puede controlar la enfermedad microscópica local y reducir el riesgo de recidivas locoregional, mientras que para otros su valor en el control de las recidivas locales del leiomiosarcoma es escaso ${ }^{7,8}$.

Siguiendo a Fagundes, aquellos tumores con diferenciación histológica grado 2 y 3 serían los más beneficiados por la radioterapia, ya que en los de grado 1 , la recidiva es menor ${ }^{8}$.

En ninguno de nuestros dos casos se llevó a cabo tratamiento adyuvante por los motivos expuestos en material y método. Uno de nuestros pacientes tuvo una recidiva local y a distancia a los 3 años de la cirugía falleciendo pocos meses después. El otro enfermo se encuentra libre de enfermedad tras 2 años de seguimiento.
Las cifras de supervivencia a cincos años son variables para aquellos pacientes diagnosticados de estas neoplasias, aceptándose un amplio rango entre el $10 \mathrm{y}$ el $62 \%{ }^{2,4,8,9}$.

\section{REFERENCIAS}

1. Castiñeiras J, Varo C, Sánchez C, Juárez A, Castro C, López A, et al. Neoplasias paratesticulares de origen mesenquimatoso. Estudio clínico-patológico. Actas Urol Esp. 1995;19(1): 40-45.

2. Llarena Ibarguren R, Azurmendi Sastre V, Martín Bazaco J, Villafruela Mateos A, Eizaguirre Zarza B, Pertusa Peña C. Leiomiosarcoma paratesticular. Revisión y puesta al día. Arch Esp Urol. 2004;57(5):525-530.

3. López A, Castiñeiras J, Vilches J, Aparicio J, Cabello P, Rodríguez-Rubio F. Rabdomiosarcoma paratesticular. Presentación de un caso y discusión de la biología de estas neoplasias. Actas Urol Esp. 1988;12(5):469-475.

4. Cruz Guerra NA, Clemente Ramos L, Montáns Araújo J, Linares Quevedo A, Pozo Mengual B, Allona Almagro A. Leiomiosarcoma paratesticular: descripción de un nuevo caso. Arch Esp Urol. 2002:55(3):311-314.

5. Rao CR, Srinivasulu M, Narres KN, Doval DC, Hazarika D. Adult paratesticular sarcomas: a report of eight cases. J Surg Oncol. 1994;56(2):89-93.

6. Costa J, Weslwy RA, Glatstein E, Rosenberg SA. The grading of soft tissue sarcomas. Results of a clinicohistopathologic correlation in a series of 163 cases. Cancer. 1984;53(3):530541.

7. Catton C, Jewett M, O’Sullivan B, Kandel R. Paratesticular sarcoma: Failure patterns after definitive local therapy. J Urol. 1999;161(6): 1844-1847.

8. Fagundes MA, Zietman AL, Althausen AF, Coen JJ, Shipley WU. The management of spermatic cord sarcoma. Cancer. 1996:77(9): 1873-1876.

9. Coleman J, Brennan MF, Alektiar K, Russo P. Adult spermatic cord sarcomas: management and results. Ann Surg Oncol. 2003;(6):669-675.

Correspondencia autor: Dr. M. Soto Delgado.

Servicio de Urología.

Hospital Universitario de Puerto Real

Ctra. Nacional IV, Km. 665 - 11510 Puerto Real (Cádiz)

Tel.: 956005000

E-mail autor: manuelsotocorreo@yahoo.es

Información artículo: Nota clínica

Trabajo recibido: abril 2006

Trabajo aceptado: octubre 2006 